\title{
Comparative study of the cardio-ankle vascular index and ankle-brachial index between young Japanese and Mongolian subjects
}

\begin{abstract}
Shuumarjav Uurtuya ${ }^{1,2}$, Nobuyuki Taniguchi ${ }^{1}$, Kazuhiko Kotani ${ }^{1}$, Toshiyuki Yamada ${ }^{1}$, Mikihiko Kawano $^{3}$, Nyamdavaa Khurelbaatar ${ }^{2}$, Kouichi Itoh ${ }^{4}$ and Tserenkhuu Lkhagvasuren ${ }^{2}$

Mongolian people have higher mortality and morbidity rates due to cardiovascular disease (CVD) than Japanese people. The cardio-ankle vascular index (CAVI) and ankle-brachial index $(\mathrm{ABI})$ are both atherosclerosis-related indexes. Presently, there is no comparative information on CAVI and ABI among young subjects between Mongolian and Japanese people. A total of one hundred Mongolian (men: 39\%, mean age: $20.9 \pm 2.2$ years) and 115 Japanese volunteers (men: $39 \%$, mean age: $22.0 \pm 1.8$ years) were recruited from among university students. The body mass index (BMI), heart rate (HR), blood pressure (BP), CAVI, $A B I$, carotid intima-media thickness, blood total cholesterol (TC), glucose and $C$ reactive protein levels were measured. The levels of BMI, HR and diastolic BP were significantly higher, and TC and glucose were significantly lower in the Mongolian subjects than in the Japanese subjects. The CAVI values (median (interquartile range): 6.5 (5.8-7.0) vs. 5.6 (5.2-6.0)) and ABI (1.11 (1.05-1.17) vs. 1.09 (1.05-1.15)) were significantly higher in the Mongolian subjects than in the Japanese subjects. The patterns of correlation between CAVI, ABI and other atherosclerotic parameters were different: in age-, gender- and BMIadjustment correlation tests for CAVI and ABI, HR ( $r=-0.25$ for CAVI and ABI) showed a correlation in the Mongolian subjects, and for ABI systolic BP $(r=-0.28)$ showed a correlation in the Japanese subjects. These results suggest that Mongolian subjects may be at higher risk of CVD, even among younger individuals, than Japanese subjects.

Hypertension Research (2009) 32, 140-144; doi:10.1038/hr.2008.28; published online 16 January 2009
\end{abstract}

Keywords: CAVI; ABI; young people; lifestyle; altitude

\section{INTRODUCTION}

Cardiovascular disease (CVD) is a major world-wide cause of premature death. ${ }^{1}$ Since 1995, CVD has been increasing and has become a more significant health issue than cancer in Mongolia. ${ }^{2}$ The WHO statistical data in 2005 suggested that the Mongolian people had a shorter life expectancy by 17 years in comparison with the Japanese people. ${ }^{3}$ In the country health information profiles of the WHO report in 2006, the mortality rates per 100000 of the population were 4.5 and 17.3 due to hypertension, 21.3 and 43.7 due to ischemic heart disease, and 246.3 and 230.6 due to cerebrovascular diseases in Japan and Mongolia, respectively. ${ }^{3}$ Therefore, comparative studies are necessary to determine the atherosclerotic factor traits between Mongolian subjects and those from countries such as Japan, which has a very high average lifespan.

One of the crucial issues to prevent CVD is the detection of atherosclerotic alterations among asymptomatic subjects. The cardio-ankle vascular index (CAVI) and ankle-brachial index (ABI) are both atherosclerosis-related indexes that are particularly reflective of arterial stiffness and stenosis, respectively. ${ }^{4,5}$ In fact, both indexes are associated with atherosclerotic risk factors and predict the future of CVD. ${ }^{4,5}$ CAVI is a new parameter, independent of blood pressure (BP), and the clinical significance of CAVI for CVD has been established recently. ${ }^{6} \mathrm{ABI}$ is also useful for the diagnosis of low extremity peripheral arterial stenotic disease. ${ }^{7}$

Age, male gender, smoking, lack of exercise, obesity, dyslipidemia, diabetes and hypertension are routinely considered to be well-known atherosclerotic risk factors. ${ }^{8,9}$ Although there have been a few comparative studies reporting higher levels of body mass index (BMI) and BP in Mongolian subjects in comparison with Japanese subjects, ${ }^{10,11}$ the earlier studies have not examined young subjects. To prevent atherosclerosis in the long term, it is necessary to start the prevention in the early stages of the condition in young people. However, no information on atherosclerosis-related parameters such as CAVI and $\mathrm{ABI}$ is available for young persons. Although the difference in lifestyle factors between Mongolian and Japanese people can also affect the status of atherosclerotic risk factors, there have been few comparative

${ }^{1}$ Department of Clinical Laboratory Medicine, Jichi Medical University, Tochigi, Japan; ${ }^{2}$ Bio-Medical School, Health Sciences University of Mongolia, Ulaanbaatar, Mongolia; ${ }^{3}$ Department of Clinical Laboratory, Jichi Medical University Saitama Medical Center, Saitama, Japan and ${ }^{4}$ Hitachi-omiya Saiseikai Hospital, Ibaraki, Japan Correspondence: Dr Sh Uurtuya, Department of Clinical Laboratory Medicine, Jichi Medical University, 3311-1 Yakushiji, Shimotsuke City, Tochigi 329-0498, Japan. E-mail: sh_uur2004@jichi.ac.jp

Received 8 September 2008; revised 23 October 2008; accepted 4 November 2008; published online 16 January 2009 
studies on regulation of lifestyle factors. Therefore, the objective of this study was to examine the atherosclerotic parameters, including CAVI and ABI, in consideration of some lifestyle factors in young Mongolian and Japanese subjects.

\section{MATERIALS AND METHODS}

A total of 100 Mongolian volunteers (men: 39, women: 61), aged 18-25 years, were recruited from the Health Sciences University of Mongolia (Ulaanbaatar city, Mongolia). Similarly, 115 volunteers (men: 45, women: 70) were recruited from the Jichi Medical University (JMU) in Tochigi Prefecture, Japan. The participants' data were collected under the same conditions (that is, during a regular class period without any examinations, or at room temperature) between June 2006 and January 2008. All eligible subjects were asymptomatic without any known history of cardiovascular, cerebrovascular, kidney or liver disease. None of the subjects were currently consuming alcohol. This study was approved by the Ethical Committee of JMU and the Mongolian Ministry of Health, and each volunteer gave informed consent.

With respect to lifestyle factors, questionnaires on meat intake (categorized by $\geqslant 4$ times in a week or $<4$ times in a week), vegetable intake ( $\geqslant 4$ times in a week or $<4$ times in a week), salt intake (high or low), smoking (current or former/never) and physical activity ( $\geqslant 1$ time in a week or $<1$ time a week) were self-administered. The BMI was calculated as weight divided by the square of body height while wearing light clothes. Serum samples were collected from the antecubital vein after a 12-h overnight fast and the samples were stored at $-80^{\circ} \mathrm{C}$. Blood measurements were performed at a laboratory facility in Japan. The serum total cholesterol and plasma glucose were measured enzymatically, and $\mathrm{C}$ reactive protein was measured using a latex agglutination immune assay (EIKEN Chemical Co. Ltd, Tokyo, Japan).

Oscillometric technology was applied to evaluate the heart rate (HR), CAVI and ABI using a VaSera VS-1000 vascular screening system (Fukuda Denshi Co. Ltd, Tokyo, Japan). The volunteers were made to lie on a bed in supine position for at least $10 \mathrm{~min}$, as described earlier. ${ }^{12}$ The CAVI values were obtained from the brachial and ankle pulse wave forms, systolic and diastolic BP (SBP and DBP), ${ }^{13}$ which were measured by using a cuff suitable for each subject's arm according to the proposed technical manual. ${ }^{14}$ The stiffness parameter was calculated using the formula CAVI $=\ln (\mathrm{Ps} / \mathrm{Pd}) \times 2 \rho / \Delta P \times \mathrm{PWV}^{2}$ (where Ps is the SBP, Pd the DBP, $\rho$ the blood density, $\Delta P$ the pulse pressure and PWV the pulse wave velocity between the aortic value and the ankle). ABI was determined based on the SBP in both the upper (brachial arterial) and lower (tibial arterial) BP. ${ }^{5} \mathrm{ABI}$ was calculated by dividing the ankle SBP by the brachial SBP. The SBP and DBP were measured for each subject's brachial artery: although this was a single measurement, the subjects showing hypertensive levels were not detected.

Moreover, after $5 \mathrm{~min}$ of relaxing in the supine position, the intima-media thickness (IMT) of the bilateral common carotid arteries of the volunteers was measured by ultrasonography (EH54-9DR system, DIASUS, Scotland, UK). The mean IMT levels were determined by the average of the values at points 1 , 2 and $3 \mathrm{~cm}$ below the carotid bifurcation on each side of artery.

\section{Statistical analysis}

The data are presented as the mean \pm s.d.; in cases of parameters with nonnormal distributions (BMI, HR, SBP, DBP, CAVI, ABI, IMT, total cholesterol, glucose and $C$ reactive protein), the data are shown as the median (interquartile range). In all analyses, parameters with non-normal distributions were used after log-transformations. All statistical analyses used the Statistical Package for Social Science (SPSS) version 16.0 for Windows (SPSS Inc., Chicago, IL, USA). A $P$-value $<0.05$ was considered to be statistically significant. Comparisons between groups were examined using Student's $t$-test (for continuous variables) and the $\chi^{2}$-test/Fisher's exact probability test (for categorical variables). As the age and BMI were significantly different between the Japanese and Mongolian subjects, age and gender were treated as co-variables in the comparison with BMI, and subsequently, in the comparison of other parameters, age, gender and BMI were treated as co-variables. To analyze the within-lifestyle-categorized group differences and between-race-based group differences in the relationship of lifestyle factors with atherosclerotic parameters, age, gender, BMI and other lifestyle factors were treated as co-variables. To investigate the correlations of
CAVI and ABI with other atherosclerotic parameters, Pearson's correlation and partial correlation tests were used with adjustments for age, gender and BMI.

\section{RESULTS}

The mean age was slightly but significantly older in the Japanese subjects than in the Mongolian subjects. In the comparative data for the atherosclerotic parameters between the young Japanese and Mongolian subjects (Table 1), the BMI in the Mongolian subjects was significantly higher than that in the Japanese subjects. The HR and DBP levels were significantly higher and SBP levels tended to be high in the Mongolian subjects. CAVI and ABI were significantly higher in the Mongolian subjects than in the Japanese subjects. The total cholesterol and glucose levels were significantly lower in the Mongolian subjects than in the Japanese subjects, but the IMT and C reactive protein levels were similar.

Regarding the correlations of CAVI and ABI to other atherosclerotic parameters in the respective race-based groups (Table 2), a simple correlation test between bivariables (model 1) showed that CAVI was positively correlated with age in the Mongolian subjects. However, a correlation test with adjustments for age, gender and BMI (model 2) clearly showed a negative correlation between CAVI and HR in the Mongolian subjects. In contrast, in both correlation models of ABI, HR was correlated negatively with ABI in the Mongolian subjects and SBP was negatively correlated with ABI in the Japanese subjects.

Regarding lifestyle factors (Table 3), the Mongolian subjects had a higher percentage of meat intake and current smoking, in comparison with that in the Japanese subjects. Other lifestyle factors did not show significant differences. As for the relationship between the lifestyle factors and atherosclerotic parameters (Table 4), both differences between the race-based groups and differences in lifestyle categories within the respective race-based groups were observed. Only vegetable and salt intake showed significant between-group differences in some atherosclerotic parameters: Mongolian subjects with lower vegetable intake levels had significantly higher levels of HR than those with higher vegetable intake levels. Japanese subjects with high salt intake had significantly higher levels of HR than those with low salt intake. In contrast, the SBP was significantly higher in subjects with low salt

Table 1 Comparison of atherosclerotic parameters between young Japanese and Mongolian subjects

\begin{tabular}{lcc}
\hline & $\begin{array}{c}\text { Japanese } \\
\text { Parameters }\end{array}$ & $\begin{array}{c}\text { Mongolian } \\
(\mathrm{n}=115)\end{array}$ \\
\hline Age (years) & $22.0 \pm 1.8$ & $20.9 \pm 2.2^{* *}$ \\
Men (\%) & 39 & 39 \\
BMI (cm/kg $)$ & $20.5(19.3-22.3)$ & $21.4(19.8-23.3)^{*}$ \\
HR (b.p.m.) & $57.0(50.3-65.0)$ & $65.0(61.0-70.0)^{* *}$ \\
SBP (mm Hg) & $111.0(105.0-120.8)$ & $114.0(108.0-121.8)$ \\
DBP (mm Hg) & $65.0(62.0-68.5)$ & $70.3(65.1-76.4)^{*}$ \\
CAVI & $5.6(5.2-6.0)$ & $6.5(5.8-7.0)^{* *}$ \\
ABI & $1.09(1.05-1.15)$ & $1.11(1.05-1.17)^{*}$ \\
IMT (mm) & $0.42(0.37-0.47)$ & $0.44(0.38-0.50)$ \\
TC (mmol I-1) & $4.40(4.04-5.06)$ & $3.99(3.55-4.38)^{* *}$ \\
Glucose (mmol I-1) & $4.88(4.61-5.16)$ & $4.22(3.90-4.50)^{* *}$ \\
CRP (mg per 100 ml) & $0.03(0.01-0.05)$ & $0.04(0.02-0.08)$ \\
\hline
\end{tabular}

Abbreviations: BMI, body mass index; HR, heart rate; SBP, systolic blood pressure; DBP, diastolic blood pressure; CAVI, cardio-ankle vascular index; ABI, ankle-brachial index; IMT, intima-media thickness; TC, total cholesterol; CRP, C reactive protein.

Only age is presented as mean \pm s.d. Other parameters are presented as the median (interquartile range).

Significance level: ${ }^{*} P<0.05,{ }^{* *} P<0.001$ (age: Student's $t$-test, gender: $\chi^{2}$-test, other parameters: Student's $t$-test after log-transformation; BMI: age and gender adjustments are performed, other parameters: age, gender and BMI adjustments are performed). 
Table 2 Correlation of the CAVI and ABI with other atherosclerotic parameters in young Japanese and Mongolian subjects

\begin{tabular}{|c|c|c|c|c|}
\hline & \multicolumn{2}{|c|}{ CAVI } & \multicolumn{2}{|c|}{$A B I$} \\
\hline & Japanese & Mongolian & Japanese & Mongolian \\
\hline Age (years) & $0.14(0.1)$ & $0.35(<0.001)^{* *}$ & $0.15(0.1)$ & $0.15(0.1)$ \\
\hline Gender (men) & $0.08(0.4)$ & $-0.12(0.2)$ & $-0.21(0.03)^{*}$ & $0.14(0.2)$ \\
\hline HR (b.p.m.) & $0.002(1.0)$ & $-0.16(0.1)$ & $-0.03(0.8)$ & $-0.23(0.02)^{*}$ \\
\hline IMT (mm) & $0.04(0.7)$ & $-0.12(0.2)$ & $-0.01(0.9)$ & $0.12(0.2)$ \\
\hline $\mathrm{TC}\left(\mathrm{mmol} \mathrm{I}^{-1}\right)$ & $0.07(0.5)$ & $0.06(0.6)$ & $0.10(0.3)$ & $0.02(0.8)$ \\
\hline Glucose $\left(\mathrm{mmol} \mathrm{I}^{-1}\right)$ & $0.02(0.8)$ & $-0.08(0.4)$ & $-0.01(1.0)$ & $0.08(0.4)$ \\
\hline CRP (mg per $100 \mathrm{ml}$ ) & $0.06(0.6)$ & $-0.16(0.1)$ & $0.11(0.3)$ & $0.16(0.1)$ \\
\hline \multicolumn{5}{|l|}{ Model 2} \\
\hline $\mathrm{TC}(\mathrm{mmol} \mathrm{I-1})$ & $0.12(0.2)$ & $0.001(1.0)$ & $0.09(0.3)$ & $-0.00(1.0)$ \\
\hline Glucose $\left(\mathrm{mmol} \mathrm{I}^{-1}\right)$ & $-0.001(0.9)$ & $-0.07(0.5)$ & $-0.002(1.0)$ & $0.06(0.6)$ \\
\hline $\mathrm{CRP}(\mathrm{mg}$ per $100 \mathrm{ml}$ ) & $0.09(0.4)$ & $0.10(0.4)$ & $-0.08(0.4)$ & $0.10(0.4)$ \\
\hline
\end{tabular}

Abbreviations: HR, heart rate; SBP, systolic blood pressure; DBP, diastolic blood pressure; ABI, ankle-brachial index; CAVI, cardio-ankle vascular index; IMT, intima-media thickness; TC, total cholesterol; CRP, C reactive protein.

Data are presented as $r$ ( $P$-value).

Model 1: simple correlation between the parameters. Model 2: partial correlation between the parameters with the adjustments for age, gender and BMI.

Significance level: ${ }^{*} P<0.05,{ }^{* *} P<0.001$.

intake than in Mongolian subjects with high salt intake. No significant relationships between CAVI, ABI and lifestyle factors were observed in either the Mongolian or the Japanese subjects.

\section{DISCUSSION}

This study is the first to compare CAVI and ABI between young Japanese and Mongolian subjects considering lifestyle factors. The overall levels of atherosclerotic parameters were shown to be higher, despite the lower levels of total cholesterol and glucose in Mongolian subjects than those in Japanese subjects. In particular, BMI, HR, DBP, CAVI and ABI levels were significantly higher in the Mongolian subjects. Although higher levels of BMI and BP in Mongolian subjects have been noted in earlier studies, ${ }^{10,11}$ those studies did not examine young people. The finding of higher levels of atherosclerotic parameters in Mongolian subjects in comparison with Japanese subjects cannot be ignored when these are present even in young subjects, if these findings are relevant to the higher rate of CVD in the Mongolian people.

In addition, the non-remarkable influences of lifestyle factors and similar levels (within reference ranges) on IMT and C reactive protein between Mongolian and Japanese subjects suggest that the present study population showed only a weak effect of their accumulated daily lifestyles, and that the subjects were free from atherosclerotic changes, probably because the population comprised young and undiseased subjects. Accordingly, the present data might be a valuable reference to observe the prospective incidence of CVD.

CAVI is a new marker for the stiffness of the aorta, femoral and tibial arteries, independently of BP. ${ }^{15}$ CAVI was higher in Mongolian subjects. Aorta stiffness was not expected in this young population, but these results imply the presence of promoting factors for arterial stiffness in Mongolian subjects. Interestingly, in the confounder-
Table 3 Comparison in lifestyle factors between young Japanese and Mongolian subjects

\begin{tabular}{|c|c|c|}
\hline Lifestyle category & Japanese & Mongolian \\
\hline \multicolumn{3}{|l|}{ Meat intake } \\
\hline$\geqslant 4$ times in a week & $15(13.0)$ & $47(47.0)^{* *}$ \\
\hline$<4$ times in a week & $100(11.5)$ & $53(53.0)$ \\
\hline \multicolumn{3}{|l|}{ Vegetable intake } \\
\hline$\geqslant 4$ times in a week & $82(71.2)$ & $80(80.0)$ \\
\hline$<4$ times in a week & $33(28.7)$ & $20(20.0)$ \\
\hline \multicolumn{3}{|l|}{ Salt intake } \\
\hline High & $14(12.2)$ & $20(20.0)$ \\
\hline Low & $101(87.8)$ & $80(80.0)$ \\
\hline \multicolumn{3}{|l|}{ Smoking } \\
\hline Current & $2(2.6)$ & $19(19.0)^{* *}$ \\
\hline Former/never & $113(97.4)$ & $81(81.0)$ \\
\hline \multicolumn{3}{|l|}{ Physical activity } \\
\hline$\geqslant 1$ time in a week & $74(64.3)$ & $62(62.0)$ \\
\hline$<1$ time in a week & $41(35.7)$ & $38(38.0)$ \\
\hline
\end{tabular}

Data are represented as number (\%).

Significance level: ${ }^{*} P<0.05,{ }^{*} P<0.001\left(\chi^{2}\right.$-test or regarding smoking only Fisher's exact probably test).

adjustment tests CAVI was significantly correlated with $\mathrm{HR}$ in Mongolian subjects, whereas a negative relationship between HR and CAVI has not been documented earlier. In contrast, ABI is shown to be a highly sensitive marker to diagnose peripheral arterial 
Table 4 Relationship between lifestyles and atherosclerotic parameters

\begin{tabular}{|c|c|c|c|c|c|c|c|}
\hline & \multicolumn{2}{|c|}{ Japanese } & \multirow[b]{2}{*}{$P_{1}$} & \multicolumn{2}{|c|}{ Mongolian } & \multirow[b]{2}{*}{$P_{2}$} & \multirow[b]{2}{*}{$P_{3}$} \\
\hline & $Q_{0}$ & $Q_{1}$ & & $Q_{0}$ & $Q_{1}$ & & \\
\hline Vegetable intake & $n=82$ & $n=33$ & & $n=80$ & $n=20$ & & \\
\hline $\mathrm{HR}$ & $57.0(50.8-65.3)$ & $57.0(49.5-63.5)$ & NS & $65.0(61.0-69.8)$ & $65.0(62.0-70.0)$ & 0.01 & $<0.0001$ \\
\hline Salt intake & $n=101$ & $n=14$ & & $n=80$ & $n=20$ & & \\
\hline HR (b.p.m.) & $56.0(49.8-64.0)$ & 65.5 (58.5-69.0) & 0.02 & $64.5(61.3-69.8)$ & $67.0(59.5-76.0)$ & NS & $<0.001$ \\
\hline $\mathrm{SBP}(\mathrm{mm} \mathrm{Hg})$ & $112.3 \pm 11.0$ & $108.9 \pm 7.3$ & NS & $114.0(108.0-123.0)$ & $115.0(106.8-119.0)$ & 0.02 & 0.02 \\
\hline
\end{tabular}

Abbreviations: $\mathrm{HR}$, heart rate; NS, no significance; SBP, systolic blood pressure. Data are presented the mean \pm s.d. or median (interquartile range). Vegetable intake: $Q_{0}=\geqslant 4$ times in a week, $Q_{1}=<4$ times in a week. Salt intake: $Q_{0}=$ less salty, $Q_{1}=$ salty.

$P_{1}$ : within-group difference in Japanese subjects. $P_{2}$ : within-group difference in Mongolian subjects. $P_{3}$ : group difference between Japanese and Mongolian subjects.

Significance level: $P<0.05$ (all analyses were performed with the adjustments for age and gender, BMI and other lifestyle factors.

stenotic disease, but it was not expected in this study population, because all values of $A B I$ did not correspond to the disease criteria level (of <0.9). ${ }^{16}$ In this situation, higher ABI levels were found in the Mongolian subjects in comparison with the Japanese subjects. The absence of a significant difference in SBP (brachial BP) between Mongolian and Japanese subjects suggests that the ankle BP might affect $\mathrm{ABI}$ levels to greater extent. Interestingly, HR was correlated negatively with $\mathrm{ABI}$ in the Mongolian subjects, which is consistent with earlier findings. ${ }^{17,18}$ In the Japanese subjects, the SBP was negatively correlated with $\mathrm{ABI}$, which is also consistent with the earlier results. ${ }^{19}$

Thus, although differences in the correlation pattern of CAVI and ABI with other atherosclerotic parameters between young Mongolian and Japanese subjects were identified, the reasons are unclear. As a possible mechanism, the residential altitude is very different between Japanese and Mongolian people and it has been documented that the altitude can contribute to circulatory characteristics such as HR and $\mathrm{BP}^{20,21}$ The mean altitude of JMU (Tochigi prefecture) is approximately $50 \mathrm{~m}$ (165 feet) and that of Ulaanbaatar is $1350 \mathrm{~m}$ (4429 feet) above sea level. In our study, the higher levels in HR and BP in the Mongolian subjects may be explained by the earlier study results that HR and BP (DBP in particular) increased with rising altitude levels. ${ }^{20,21}$ However, basically CAVI is independent of BP (at least, the direct causes of increases in BP cannot necessarily be similar to those in CAVI). Importantly, one study has suggested that CAVI levels are higher in residents living at high altitude than those at sea level, and increases in hematocrit and oxidative stress by hypoxia are speculated as the causes. ${ }^{21}$ In fact, the increased hematocrit levels in Mongolian subjects have already been reported. ${ }^{20}$ Therefore, the results of higher CAVI levels in the Mongolian subjects may be partly due to such hypoxia-related factors. In addition, the higher ABI levels obtained in the Mongolian subjects may be partly explained by the fact that sympathetic nerve activity (in daily life) at high altitude also increases peripheral $\mathrm{BP}^{22}$ leading to a relative increase in the ankle $\mathrm{BP}$ to brachial BP. Therefore, the characteristics of atherosclerotic parameters in Mongolian subjects could be associated with the altitude level at which they live.

Moreover, although the genetic backgrounds are generally thought to be similar between Mongolian and Japanese people, ${ }^{23}$ the frequencies of gene polymorphisms might be partly different. ${ }^{24,25}$ For instance, a similar frequency of Japanese $(8.8 \%)$ and Mongolian (9.9\%) people having the Di (a) antigens is called the Mongoloid factor, ${ }^{23}$ but some of the gene polymorphisms are different (for example, apolipoprotein $\varepsilon 4$ allele and interleukin 8-251 A/T polymorphism). ${ }^{24,25}$ Therefore, undetermined genetic factors contributing to the atherosclerotic parameters, especially CAVI and ABI, might explain some of the results.
This study had an advantage in that all measurements were performed using the same methodology and at the same facility, which seems to be important in comparative studies between countries. There are also a few limitations. Although the questionnaire generally covered the representative lifestyles relating to atherosclerosis, the categorization was simple and the detailed content of lifestyles was not confirmed. This could lead to difficulty in detecting the influences of lifestyle factors on atherosclerotic parameters. Although the sample size was small, the fact that the study population consisted of persons who got into the universities at a nationwide level in the respective countries strengthened the generalization of the results. The measures of hematocrit level, oxidative stress, sympathetic nerve activity and genetic polymorphisms were not examined, although these measures may provide further information on the altitudeatherosclerotic connection. Therefore, a study with a larger sample size and more detailed lifestyle-related questionnaires and the measurements of various biomarkers is a future challenge. Furthermore, other undetermined factors, for example, environmental factors other than altitude, should also be considered.

In conclusion, this study found that CAVI and ABI, in addition to BMI, HR and DBP, were significantly higher in the Mongolian subjects than in the Japanese subjects. These findings suggest that Mongolian people may be at higher risk of CVD, even among the younger generation. Further studies are needed to clarify the bio-physiological mechanism of higher levels of atherosclerotic parameters.

\section{ACKNOWLEDGEMENTS}

This study was in part supported by a grant-in-aid from the Japanese Society of Laboratory Medicine Fund for the Promotion of Scientific Research.

1 World Health Organization. Preventing Chronic Disease: A Vital Investment. WHO: Geneva, 2005

2 Annual Health Report 2006 Ministry of Health National Center for Health Development (in Mongolia), 2006, pp 35.

3 Country Health Information Profiles. 2006 Revision. WHO: Western pacific region, 2006.

4 Nakamura K, Tomaru T, Yamamura S, Miyashita Y, Shirai K, Noike H. Cardio-ankle vascular index is a candidate predictor of coronary atherosclerosis. Circ $J$ 2008; 72 : 598-604.

5 Price JF, Tzoulaki I, Lee AJ, Fowkes FG. Ankle brachial index and intima-media thickness predict cardiovascular events similarly and increased prediction when combined. J Clin Epidemol 2007; 60: 1067-1075.

6 Ibata J, Sasaki H, Kakimoto T, Matsuno S, Nakatani M, Kobayashi M, Tatsumi K, Nakano Y, Wakasaki H, Furuta H, Nishi M, Nanjo K. Cardio-ankle vascular index measures arterial wall stiffness independent of blood pressure. Diabetes Res Clin Pract 2008; 80: 265-270.

7 Bernestein EF, Fronek A. Current status of non-invasive tests in the diagnosis of peripheral arterial disease. Surg Clin North Am 1982; 62: 473-487. 
8 Joseph L, Izzo JR, Sica DA, Blabk HR. Hypertension Primer. Atherosclerosis and coronary disease. Lippincott Williams \& Wilkins: USA, 2008, pp 209-213.

9 Wilson PW, Evans JC. Coronary artery disease prediction. Am J Hypertens 1993; 6: S309-S313.

10 Shiwaku K, Anuurad E, Enkhmaa B, Nogi A, Kitajima K, Shimono K, Yamane Y, Oyunsuren T. Overweight Japanese with body mass indexes of 23.0-24.9 have higher risks for obesity-associated disorders: a comparison of Japanese and Mongolians. Int J Obes 2004; 28: 152-158.

11 Anuuraad E, Shiwaku K, Nogi A. Ethnic differences in the formation of small LDL particles in Asians: a comparison of Koreans, Japanese and Mongolians. Eur J Clin Invest 2004; 34: 738-746.

12 Okura T, Watanabe S, Kurata M, Manabe S, Koresawa M, Irita J, Enomoto D, Miyoshi K, Fukuoka T, Higaki J. Realtionship between cardio-ankle vascular index (CAVI) and carotid atherosclerosis in patients with essential hypertension. Hypertens Res 2007; 30: 335-340.

13 Shirai K, Utino J, Otsuka K, Takata M. A novel blood pressure-independent arterial wall stiffness parameters; Cadio-Ankle Vacular Index (CAVI). J Atheroscler Thromb 2006; 13: 101-107.

14 Operation manual of the Vasera VS-1000 Fukuda Denshi UK; Tokyo, Japan, pp 71 2005 (in Japanese).

15 Akira $\mathrm{Y}$, Makoto H, Yayoi N, Yamada A, Hayakawa M, Nakayama Y, Amimoto K, Yoshinaga $Y$, Kagawa $K$, Takaki A, Ogawa $H$. The basic clinical assessment of cardio vascular index. Japanese J Med Technol 2006; 55: 120-125.

16 Yao ST, Hobbs JT, Irvine WT. Ankle systolic pressure measurements in arterial disease affecting the lower extremities. Br J Surg 1969; 56: 676-679.

17 Su H-M, Lee K-T, Chu C-S, Lee M-Y, Lin T-H, Voon W-C, Sheu S-H, Lai W-T. Effects of heart rate on brachial-ankle pulse wave velocity and ankle-brachial pressure index in patients without significant organic heart disease. Angiology 2007; 58: 67-74.
18 Abraham P, Desvaux B, Colin D, Leftheriotis G, Saumet J-L. Heart rate- corrected ankleto-arm index in the diagnosis of moderate lower extremity arterial disease. Angiology 1995; 46: 673-677.

19 Male S, Coull A, Murphy-Black T. Preliminary study to investigate the normal range of ankle brachial pressure index in young adults. J Clin Nurs 2007; 6: 1878-1885.

20 Olziikhutag A. Adaptation of aboriginal inhabitants and regional pathology in condition of Mongolian median altitude. Ulaanbaatar: Mongolia, 2000, pp. 299-317.

21 Otsuka K, Norboo T, Otsuka Y, Higuchi H, Hayajiri M, Narushima C, Sato Y, Tsugoshi T, Murakami S, Wada T, Ishine M, Okumiya K, Matsubayashi K, Yano S, Chogyal T, Angchuk D, Ichihara K, Cornélissen G, Halberg F. Chronoecological health watch of arterial stiffness and neuro-cardio-pulmonary function in elderly community at high altitude (3524m), compared with Japanese town. Biomed Pharmacother 2005; 59: S58-S67.

22 Schneider A, Greene RE, Keyl C, Bandinelli G, Passino C, Spadacini G, Bonfichi M, Arcaini L, Malcovati L, Boiardi A, Feil P, Bernardi L. Peripheral arterial vascular function at altitude: sea-level natives versus Himalayan high-altitude natives. J Hypertens 2001; 19: 213-222.

23 Komatsu F, Hasegawa K, Yanagisawa Y, Kawabata T, Kaneko Y, Watanabe S, Miyagi S, Sakuma M, Kagawa Y, Kajiwara M. Prevalence of Diego Blood Group Dia antigen in Mongolians: Comparison with that in Japanese. Transfus Apher Sci 2004; 30 . 119-124.

24 Svobodová H, Kucera F, Stulc T, Vrablík M, Amartuvshin B, Altannavch TS, Ceska R. Apolipoprotein E gene polymorphism in the Mongolian population. Foli Biologica 2007; 53: 138-142.

25 Fujihara J, Shiwaku K, Yasuda T, Yuasa I, Nishimukai H, lida R, Takeshita H. Variation of interleukin 8 -251 A>T polymorphism in worldwide populations and intra-ethnic differences in Japanese population. Clin Chim Acta 2007; 377: 79-82. 